\title{
Synthesis, Characterization and Evaluation of Anti-inflammatory Activity of Novel Indoline Derivatives
}

\author{
Dhineshkumar Manoharan ${ }^{\mathrm{a}^{*}}$, Kannan Kulanthai ${ }^{\mathrm{a}}$ and Gnanavel Sadhasivam ${ }^{\mathrm{a}}$ \\ $a^{*}$ Department of chemistry, Government College of Engineering, Salem-636011, Tamil Nadu, India. \\ ${ }^{a}$ Department of chemistry, Government College of Engineering, Salem-636011, Tamil Nadu, India. \\ ‘E-mail: dhineshkavi2020@gmail.com, Phone: +919787809030
}

\begin{abstract}
Series of indoline derivatives were synthesized using $\mathrm{N}$-(4-aminophenyl) indoline-1-carbothiamide as a precursor. The structures of synthesized compounds were confirmed by $\mathrm{FT}-\mathrm{IR},{ }^{1} \mathrm{H}-\mathrm{NMR},{ }^{13} \mathrm{C}-\mathrm{NMR}$ and LC-MS. The in vitro antiinflammatory activity of synthesized indoline derivatives were examined by standard anti-denaturation assay. The compounds $4 \mathrm{a}\left(\mathrm{IC}_{50}=62.2 \mu \mathrm{g} / \mathrm{ml}\right)$ and $4 \mathrm{~b}\left(\mathrm{IC}_{50}=60.7 \mu \mathrm{g} / \mathrm{ml}\right)$ showed potent inhibition on protein denaturation. The compounds $5 \mathbf{a}\left(\mathrm{IC}_{50}=97.8 \mu \mathrm{g} / \mathrm{ml}\right)$ exhibits moderate inhibition on protein denaturation.
\end{abstract}

Keywords: indoline, anti-inflammatory, anti-denaturation, ${ }^{1} \mathrm{H}-\mathrm{NMR}$.

\section{Introduction}

Heterocyclic compounds containing five or six membered ring are essential for their various biological activities [1] Indoline the potent basic pharmacodynamic nucleus, has been reported to possess a wide variety of biological properties such as antimicrobial [2-5], anticancer [6-10], anti-diabetic [11], anti-inflammatory [12-13], antitubulin[14], antihyperlipidemic [15], anti-atherosclerotic [15], anticoagulant [16], tyrosinekinase inhibitor [17], antiperoxidative [18], antioxidant [19], anti-hepatocellularcarcinoma [20], Antimitoic [21] and Antagonists[22-23].

In addition, the indoline nucleus is incorporated in various natural products such as alkaloids [24]. Encouraged by the above observations and considering the interesting pharmacological profile of indoline, we synthesized $\mathrm{N}$-(4aminophenyl)indoline-1-carbothioamide scaffold based compounds as anti-inflammatory agents. The biological activity and structure-activity relationship (SARs) of the newly synthesized indoline derivatives discussed.

\section{EXPERIMENTAL}

\subsection{Chemistry}

The synthetic starting materials such as reagents and solvents were used in analytical grade or the highest quality commercially available and were purchased from Sigma-Aldrich Chemical Co., Merck Chemical Co. Melting points were recorded by labtronics digital melting points apparatus. The ${ }^{1} \mathrm{H}-\mathrm{NMR}$ and ${ }^{13} \mathrm{C}-\mathrm{NMR}$ spectra were recorded in $\mathrm{DMSO} \mathrm{- \textrm {d } _ { 6 }}$ solvent on Bruker $300 \mathrm{MHz}$ spectrophotometer using tetramethylsilane as an internal reference. The apparent resonance multiplicity is described as $\mathrm{s}$ (singlet), $\mathrm{d}$ (doublet), dd (doublet of doublet), $\mathrm{t}$ (triplet), td (triplet of doublet), $\mathrm{q}$ (quartet) and $\mathrm{m}$ (multiplet). Infrared measurements were recorded in the range $400-4000 \mathrm{~cm}^{-1}$ by Perkin Elmer. Mass spectra were recorded on a Thermo LCQ Deca XP MAX at $70 \mathrm{eV}$. Thin layer chromatography (TLC) analysis was carried out on $5 \times 20$ $\mathrm{cm}$ plate coated with silica gel $\mathrm{GF}_{254}$.

\subsubsection{Synthesis of $\mathrm{N}$-(4-nitrophenyl) indoline-1-carbothioamide (1)}

To a solution of indoline $(10 \mathrm{~g}, 0.0835 \mathrm{~mol})$ in $200 \mathrm{~mL}$ of THF, 4-nitrophenyl isothiocyanate $(16.53 \mathrm{~g}, 0.0918 \mathrm{~mol})$ was added at $0^{\circ} \mathrm{C}$. The resulting reaction mass was allowed to $\mathrm{RT}$ and stirred for $4 \mathrm{hr}$. The reaction mass was concentrated under reduced pressure to give the compound 1 as a yellow solid; Yield 80\%; m.p 173-174 ${ }^{\circ} \mathrm{C}$; IR $(\mathrm{KBr}) \mathrm{V}_{\max }$ in $\mathrm{cm}^{-1}$ : 3434, 3300 (NH stretch), 3074 (CH stretch, aromatic), 2919, 2850 (CH stretch, aliphatic), 1532, 1319, ( $\mathrm{NO}_{2}$ stretch), 1477 $\left(\mathrm{CH}_{2}\right.$ bend), $1270(\mathrm{C}=\mathrm{S}), 909$ (C-N stretch) ; ${ }^{1} \mathrm{H}-\mathrm{NMR}\left(\mathrm{DMSO}_{6}\right) \delta \mathrm{ppm}: 3.13(\mathrm{t}, 2 \mathrm{H}, \mathrm{J}=8.1 \mathrm{~Hz}), 4.32(\mathrm{t}, 2 \mathrm{H}, \mathrm{J}=8.2 \mathrm{~Hz})$, $7.05(\mathrm{td}, 1 \mathrm{H}, J=0.9,7.9 \mathrm{~Hz}), 7.18(\mathrm{td}, 1 \mathrm{H}, J=1.2,8.1 \mathrm{~Hz}), 7.33(\mathrm{~d}, 1 \mathrm{H}, J=7.9 \mathrm{~Hz}), 7.71(\mathrm{~d}, 2 \mathrm{H}, J=9.9 \mathrm{~Hz}), 7.80(\mathrm{~d}, 1 \mathrm{H}$, $J=8.0 \mathrm{~Hz}), 8.19(\mathrm{~d}, 2 \mathrm{H}, J=9.9 \mathrm{~Hz}), 10.39(\mathrm{~s}, 1 \mathrm{H}) ;{ }^{13} \mathrm{C}-\mathrm{NMR}$ (DMSO-d $\left.\mathrm{d}_{6}\right) \delta \mathrm{ppm}: 26.6,54.2,116.0,121.9,123.9,124.1$, 125.6, 126.8, 134.3, 142.1, 142.2, 146.8, 177.5; LC-MS calculated for $\left(\mathrm{C}_{15} \mathrm{H}_{13} \mathrm{~N}_{3} \mathrm{O}_{2} \mathrm{~S}\right) \mathrm{m} / \mathrm{z}[\mathrm{M}+\mathrm{H}]^{+} 299.07$, found m/z 300.0665

\subsubsection{Synthesis of $\mathrm{N}$-(4-aminophenyl) indoline-1-carbothioamide (2)}

To a solution of compound 1 (10 g, $0.0338 \mathrm{~mol})$ in $100 \mathrm{~mL} 12 \mathrm{~N} \mathrm{HCl}, \mathrm{SnCl}_{2}(63.88 \mathrm{~g}, 0.338 \mathrm{~mol})$ was added at RT. The resulting reaction mass was stirred for $3 \mathrm{hr}$. The completion of the reaction was monitored by TLC. The reaction mass was diluted with $350 \mathrm{~mL}$ of cold water. The resulting reaction mass was basified to $\mathrm{pH} 8$ with $40 \% \mathrm{NaOH}$. The aqueous layer was extracted with ethyl acetate $(3 \times 150 \mathrm{~mL})$ and water $(2 \times 30 \mathrm{~mL})$, brine $(1 \times 300 \mathrm{~mL})$ and dried over anhydrous $\mathrm{Na}_{2} \mathrm{SO}_{4}$. The organic layer was concentrated under reduced pressure to afford compound 2. Brown solid; Yield $72 \% ; \mathrm{mp}$ 209-210 ${ }^{\circ} \mathrm{C}$; IR (KBr) $v_{\max }$ in $\mathrm{cm}^{-1}$ : 3398 (NH stretch), 3055 (CH stretch, aromatic), 2969 (CH stretch, aliphatic), 1610 (NH bend), $1447\left(\mathrm{CH}_{2}\right.$ bend), $1209(\mathrm{C}=\mathrm{S}), 932$ (C-N stretch) ; ${ }^{1} \mathrm{H}-\mathrm{NMR}$ (DMSO-d $)$ ס ppm: $3.08(\mathrm{t}, 2 \mathrm{H}, \mathrm{J}=8.1 \mathrm{~Hz}), 4.21$ (t, $2 \mathrm{H}$, $J=8.2 \mathrm{~Hz}), 5.05(\mathrm{~s}, 2 \mathrm{H}), 6.52(\mathrm{~d}, 2 \mathrm{H}, J=8.7 \mathrm{~Hz}), 6.95(\mathrm{t}, 3 \mathrm{H}, J=7.2 \mathrm{~Hz}), 7.12(\mathrm{t}, 1 \mathrm{H}, J=7.8 \mathrm{~Hz}), 7.24(\mathrm{~d}, 1 \mathrm{H}, J=7.9$ $\mathrm{Hz}$ ), $8.29(\mathrm{~d}, 1 \mathrm{H}, J=8.1 \mathrm{~Hz}), 9.41(\mathrm{~s}, 1 \mathrm{H}) ;{ }^{13} \mathrm{C}-\mathrm{NMR}$ (DMSO-d $)$ $\delta \mathrm{ppm}: 26.7,52.8,113.3,116.1,122.5,124.9,126.1$, 126.8, 128.5, 133.3, 143.4, 146.3, 178.5; LC-MS calculated for $\left(\mathrm{C}_{15} \mathrm{H}_{15} \mathrm{~N}_{3} \mathrm{~S}\right) \mathrm{m} / \mathrm{z}[\mathrm{M}+\mathrm{H}]^{+} 269.100$, found $\mathrm{m} / \mathrm{z} 270.0979$.

\subsubsection{Synthesis of 1-(4-fluorophenyl)-3-(4-(indoline-1-carbothioamido)phenyl)thiourea (3a)}


To a solution of compound $2(0.4 \mathrm{~g}, 0.0015 \mathrm{~mol})$ in $12 \mathrm{~mL}$ THF, 4-fluoro phenyl isothiocyanate $(0.300 \mathrm{~g}, 0.0019 \mathrm{~mol})$ was added at $0^{\circ} \mathrm{C}$. The reaction mixture was allowed to RT and stirred for $2 \mathrm{hr}$. The progress of the reaction was monitored by TLC. The reaction mass was concentrated under reduced pressure and separated between ethyl acetate $(1 \times 50 \mathrm{~mL})$ and $2 \mathrm{~N} \mathrm{HCl}(1 \times 25 \mathrm{~mL})$. The resulting ethyl acetate layer was washed with brine $(1 \times 40 \mathrm{~mL})$ and dried over anhydrous $\mathrm{Na}_{2} \mathrm{SO}_{4}$. The ethyl acetate layer was concentrated under reduced pressure to afford compound 3a. Pal yellow solid; Yield $32 \%$; mp 259-261 ${ }^{\circ} \mathrm{C}$; IR ( $\left.\mathrm{KBr}\right) \mathrm{v}_{\max }$ in $\mathrm{cm}^{-1}: 3424$ (NH stretch), 2926 (CH stretch, aromatic), 2581 (CH stretch, aliphatic), 1590 (NH bend), 1449 ( $\mathrm{CH}_{2}$ bend), 1291 (C-N stretch), 1211 (C=S), 761 (CH bend); ${ }^{1} \mathrm{H}-\mathrm{NMR}$ (DMSO-d $\left.\mathrm{d}_{6}\right) \delta \mathrm{ppm:} 3.10$ (t, $2 \mathrm{H}, J=8.3 \mathrm{~Hz}$ ), $4.26(\mathrm{t}, 2 \mathrm{H}, J=8.1 \mathrm{~Hz}), 6.99$ (t, $1 \mathrm{H}, J=7.8 \mathrm{~Hz}), 7.13-7.15(\mathrm{~m}, 3 \mathrm{H}), 7.29(\mathrm{~d}, 1 \mathrm{H}, J=8.3 \mathrm{~Hz}), 7.27-7.50$ $(\mathrm{m}, 6 \mathrm{H}), 8.09(\mathrm{~d}, 1 \mathrm{H}, J=8.4 \mathrm{~Hz}), 9.76(\mathrm{~s}, 3 \mathrm{H}) ;{ }^{13} \mathrm{C}-\mathrm{NMR}\left(\mathrm{DMSO}-\mathrm{d}_{6}\right) \delta \mathrm{ppm}: 26.7,53.3,114.8,115.1,123.0,123.6,125.2$, 126.0, 126.4, 133.7, 135.7, 143.0, 157.8, 160.2,178.2, 179.9; LC-MS calculated for $\left(\mathrm{C}_{22} \mathrm{H}_{19} \mathrm{FN}_{4} \mathrm{~S}_{2}\right) \mathrm{m} / \mathrm{z}[\mathrm{M}+\mathrm{H}]^{+} 422.1$, found $\mathrm{m} / \mathrm{z} 423.0681$

\subsubsection{Synthesis of 1-(4-(indoline-1-carbothioamido) phenyl)-3-(3-methoxy phenyl)urea (3b)}

To a solution of compound $2(0.5 \mathrm{~g}, 0.0018 \mathrm{~mol})$ in $15 \mathrm{~mL}$ THF, 3-methoxy phenyl isocynate $(0.322 \mathrm{~g}, 0.0024$ mol) was added at $0^{\circ} \mathrm{C}$. The reaction was carried out at RT and stirred for $1 \mathrm{hr}$. The completion of reaction was monitored by TLC. To the resulting residue, diethyl ether was added and the solid was filtered off. The resulting white solid was dried under vacuum to afford compound $\mathbf{3 b}$. Yield 76\%; mp 246-248 ${ }^{\circ} \mathrm{C} ; \mathrm{IR}(\mathrm{KBr}) \mathrm{v}_{\max }$ in $\mathrm{cm}^{-1}: 3287(\mathrm{NH}$ stretch), $2950(\mathrm{CH}$ stretch, aromatic), 2838 ( $\mathrm{CH}$ stretch, aliphatic), 1649 (C=O), 1558 ( $\mathrm{NH}$ bend), $1491\left(\mathrm{CH}_{2}\right.$ bend), 1291 (C-N amide), 1230 $(\mathrm{C}=\mathrm{S}), 1160$ (C-O), 763 (CH bend); ${ }^{1} \mathrm{H}-\mathrm{NMR}$ (DMSO-d $) \delta$ ppm: 3.10 (t, $\left.2 \mathrm{H}, J=8.1 \mathrm{~Hz}\right), 3.70(\mathrm{~s}, 3 \mathrm{H}), 4.27(\mathrm{t}, 2 \mathrm{H}, J=8.1$ $\mathrm{Hz}), 6.52(\mathrm{dd}, 1 \mathrm{H}, J=2.1,8.1 \mathrm{~Hz}), 6.92-7.00(\mathrm{~m}, 2 \mathrm{H}), 7.11-7.27(\mathrm{~m}, 6 \mathrm{H}), 7.43(\mathrm{~d}, 2 \mathrm{H}, J=8.7 \mathrm{~Hz}), 8.23(\mathrm{~d}, 1 \mathrm{H}, J=8.2 \mathrm{~Hz})$, $9.41(\mathrm{~d}, 2 \mathrm{H}, J=9.1 \mathrm{~Hz}), 9.67(\mathrm{~s}, 1 \mathrm{H}) ;{ }^{13} \mathrm{C}-\mathrm{NMR}\left(\mathrm{DMSO}-\mathrm{d}_{6}\right) \delta \mathrm{ppm:} 26.7,53.1,54.8,103.5,106.8,110.1,116.1,117.6$, $122.8,125.0,125.8,126.2,129.4,133.6,136.9,141.2,141.2,143.2,152.7,159.6,178.4$; LC-MS calculated for $\left(\mathrm{C}_{23} \mathrm{H}_{22} \mathrm{~N}_{4} \mathrm{O}_{2} \mathrm{~S}\right) \mathrm{m} / \mathrm{z}[\mathrm{M}+\mathrm{H}]^{+} 418.15$, found $\mathrm{m} / \mathrm{z} 419.1155$.

\subsubsection{Synthesis of $\mathbf{N}$-(4-(tosylamino) phenyl) indoline-1-carbothio amide (4a)}

To a solution of compound $2(0.4 \mathrm{~g}, 0.00150 \mathrm{~mol})$ in $10 \mathrm{~mL}$ THF, pyridine $(0.474 \mathrm{~mL}, 0.006 \mathrm{~mol})$ was added at $0^{\circ} \mathrm{C}$ followed by 4-toluene sulfonyl chloride $(0.285 \mathrm{~g}, 0.00150 \mathrm{~mol})$ in $2 \mathrm{~mL}$ 1,2-dichloroethane was added. The reaction mixture was allowed to RT and stirred for $3 \mathrm{hr}$. The progress of the reaction was monitored by TLC. The reaction mixture was concentrated under reduced pressure and separated between ethyl acetate $(50 \mathrm{~mL})$ and water $(50 \mathrm{~mL})$. The combined ethyl acetate layer was washed with $2 \mathrm{~N} \mathrm{HCl}(1 \times 30 \mathrm{~mL}), 10 \% \mathrm{NaHCO}_{3}(2 \times 25 \mathrm{~mL})$, brine $(2 \times 50 \mathrm{~mL})$ and dried over anhydrous $\mathrm{Na}_{2} \mathrm{SO}_{4}$. The ethyl acetate layer was concentrated under reduced pressure to afford compound 4a. White solid; Yield $48 \% \mathrm{mp} 220-222^{\circ} \mathrm{C}$; IR (KBr) $\mathrm{v}_{\max }$ in $\mathrm{cm}^{-1}: 3423$ (NH stretch), 3282, 3059 (CH stretch, aromatic), 2921 (CH stretch, aliphatic), 1595 ( $\mathrm{NH}$ bend), $1516\left(\mathrm{CH}_{2}\right.$ bend), 1371, $1154(\mathrm{~S}=\mathrm{O}), 749\left(\mathrm{CH}\right.$ bend); ${ }^{1} \mathrm{H}-\mathrm{NMR}\left(\mathrm{DMSO}-\mathrm{d}_{6}\right) \delta \mathrm{ppm}:$ $2.34(\mathrm{~s}, 3 \mathrm{H}), 3.08(\mathrm{t}, 2 \mathrm{H}, J=8.4 \mathrm{~Hz}), 4.22(\mathrm{t}, 2 \mathrm{H}, J=8.4 \mathrm{~Hz}), 6.95-7.09(\mathrm{~m}, 3 \mathrm{H}), 7.11(\mathrm{t}, 1 \mathrm{H}, J=7.8 \mathrm{~Hz}), 7.20-7.27(\mathrm{~m}$ $3 \mathrm{H}), 7.35(\mathrm{~d}, 2 \mathrm{H}, J=8.1 \mathrm{~Hz}), 7.64(\mathrm{~d}, 2 \mathrm{H}, J=8.1 \mathrm{~Hz}), 8.9(\mathrm{~d}, 1 \mathrm{H}, J=8.1 \mathrm{~Hz}), 9.63(\mathrm{~s}, 1 \mathrm{H}), 10.18(\mathrm{~s}, 1 \mathrm{H}) ;{ }^{13} \mathrm{C}-\mathrm{NMR}$ (DMSO-d ( $\delta$ ppm: 20.9, 26.6, 53.1, 116.0, 120.1, 123.0, 125.1, 125.66, 126.3, 126.6, 129.6, 133.6, 134.2, 136.2, 136.7, 142.8, 143.1, 178.1; LC-MS calculated for $\left(\mathrm{C}_{22} \mathrm{H}_{21} \mathrm{~N}_{3} \mathrm{O}_{2} \mathrm{~S}_{2}\right) \mathrm{m} / \mathrm{z}[\mathrm{M}+\mathrm{H}]^{+} 423.11$, found $\mathrm{m} / \mathrm{z} 424.0771$

\subsubsection{Synthesis of $\mathrm{N}-(4-\{[(2,6-$ dichlorophenyl)sulfonyl ]amino\}phenyl) indoline-1-carbothio amide (4b)}

Prepared as reported above for $4 \mathrm{a}$, starting from compound 2 and 2,6-dichlorobenzene-1-sulfonyl chloride. This reaction was carried out at room temperature for $3 \mathrm{hr}$. Red solid; Yield $37 \% ; \mathrm{mp} 215-217^{\circ} \mathrm{C} ; \mathrm{IR}(\mathrm{KBr}) \mathrm{V}_{\max }$ in $\mathrm{cm}^{-1}: 3432$ (NH stretch), 3360, 3099 (CH stretch, aromatic), 2922, 2850 (CH stretch, aliphatic), 1600 ( $\mathrm{NH}$ bend), $1519\left(\mathrm{CH}_{2}\right.$ bend), 1387, 1165 (S=O), 747 (CH bend); ${ }^{1} \mathrm{H}-\mathrm{NMR}$ (DMSO-d $\mathrm{d}_{6} \delta \mathrm{ppm}: 3.09$ (t, $2 \mathrm{H}, J=8.1 \mathrm{~Hz}$ ), 4.21 (t, $2 \mathrm{H}, J=8.2 \mathrm{~Hz}$ ), 7.04-7.07 $(\mathrm{m}, 2 \mathrm{H}), 7.14(\mathrm{~d}, 1 \mathrm{H}, J=8.5 \mathrm{~Hz}), 7.23-7.26(\mathrm{~m}, 3 \mathrm{H}), 7.52-7.71(\mathrm{~m}, 3 \mathrm{H}), 8.10(\mathrm{~d}, 1 \mathrm{H}, J=8.4 \mathrm{~Hz}), 9.63(\mathrm{~s}, 1 \mathrm{H}), 10.78(\mathrm{~s}, 1 \mathrm{H})$; ${ }^{13}$ C-NMR (DMSO- $\left.d_{6}\right) \quad \delta$ ppm: 27.1, 53.7, 116.5, 119.6, 123.5, 125.6, 126.3, 126.8, 127.6, 130.7, 132.4, 133.7, 134.2, 134.92, 136.8, 143.4,178.7 ; LC-MS calculated for $\left(\mathrm{C}_{21} \mathrm{H}_{17} \mathrm{Cl}_{2} \mathrm{~N}_{3} \mathrm{O}_{2} \mathrm{~S}_{2}\right) \mathrm{m} / \mathrm{z}[\mathrm{M}+\mathrm{H}]^{+} 477.01$, found $\mathrm{m} / \mathrm{z} 477.9745$

\subsubsection{Synthesis of 2-(1H-indol-3-yl)-N-(4-(indoline-1-carbothio amido) phenyl) acetamide (5a)}

To a solution of compound $2(0.4 \mathrm{~g}, 0.00150 \mathrm{~mol})$ in $12 \mathrm{~mL}$, 3-indole acetic acid $(0.316 \mathrm{~g}, 0.00181 \mathrm{~mol})$ EDC. HCl $(0.370 \mathrm{~g}, 0.00195 \mathrm{~mol})$ and HOBt $(0.202 \mathrm{~g}, 0.00150 \mathrm{~mol})$ was added. The mixture was cooled to $0^{\circ} \mathrm{C}$ and triethylamine $(0.818 \mathrm{~mL}, 0.006 \mathrm{~mol})$ was added. The resulting solution was allowed to RT and stirred for $8 \mathrm{hr}$. The reaction was monitored by TLC. The reaction mixture was concentrated under reduced pressure and resulting crude product was separated between ethyl acetate $(50 \mathrm{~mL})$ and water $(50 \mathrm{~mL})$. The combined ethyl acetate layer was washed with $2 \mathrm{~N} \mathrm{HCl}$ $(1 \times 30 \mathrm{~mL}), 10 \% \mathrm{NaHCO}_{3}(2 \times 20 \mathrm{~mL})$, brine $(2 \times 50 \mathrm{~mL})$ and dried over anhydrous $\mathrm{Na}_{2} \mathrm{SO}_{4}$. The organic layer was concentrated under reduced pressure to afford compound 5a. Pal yellow solid; Yield $40 \%$; $\mathrm{mp} 243-245^{\circ} \mathrm{C}$; IR $(\mathrm{KBr}) \mathrm{V}_{\max }$ in $\mathrm{cm}^{-1}$ : 3406 (NH stretch), 3310, 3184 (CH stretch, aromatic), 2904 (CH stretch, aliphatic), 1657 (C=O), 1527 (NH bend), $1482\left(\mathrm{CH}_{2}\right.$ bend), 1291 (C-N), $1254(\mathrm{C}=\mathrm{S}), 788$ (CH bend); ${ }^{1} \mathrm{H}-\mathrm{NMR}$ (DMSO-d $\left.\mathrm{d}_{6}\right) \delta \mathrm{ppm}: 3.09$ (t, $2 \mathrm{H}, \mathrm{J}=8.1 \mathrm{~Hz}$ ), $3.72(\mathrm{~s}$, 2H), $4.24(\mathrm{t}, 2 \mathrm{H}, J=8.1 \mathrm{~Hz}), 6.97(\mathrm{td}, 2 \mathrm{H}, J=1.2,6.9 \mathrm{~Hz}), 7.0-7.15(\mathrm{~m}, 2 \mathrm{H}), 7.28(\mathrm{~m}, 4 \mathrm{H}), 7.35(\mathrm{~d}, 1 \mathrm{H}, J=8.1 \mathrm{~Hz}), 7.54-$ $7.62(\mathrm{~m}, 3 \mathrm{H}), 8.16(\mathrm{~d}, 1 \mathrm{H}, J=8.3 \mathrm{~Hz}), 9.67(\mathrm{~s}, 1 \mathrm{H}), 10.12(\mathrm{~s}, 1 \mathrm{H}), 10.92(\mathrm{~s}, 1 \mathrm{H}) ;{ }^{13} \mathrm{C}-\mathrm{NMR}$ (DMSO-d $)_{6} \delta \mathrm{ppm}: 26.7,33.7$, 53.1, 108.6, 111.35, 116.0, 118.3, 118.6, 118.9, 120.9, 122.9, 123.8, 125.1, 126.3, 127.2, 133.6, 135.0, 136.1, 136.2, 143.1, 169.5, 178.4 ; LC-MS calculated for $\left(\mathrm{C}_{25} \mathrm{H}_{22} \mathrm{~N}_{4} \mathrm{OS}\right) \mathrm{m} / \mathrm{z}[\mathrm{M}+\mathrm{H}]^{+} 426.15$, found $\mathrm{m} / \mathrm{z} 427.1205$.

\subsubsection{Synthesis of 3-fluoro-N-(4-(indoline-1-carbothio amido)phenyl)benzamide (5b)}




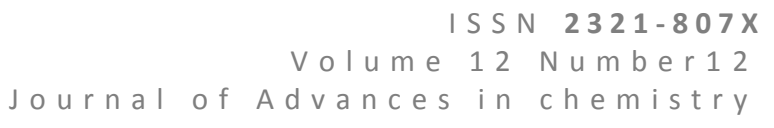

Prepared as reported above for $5 \mathrm{a}$, starting from compound 2 and 3 -fluorobenzoic acid. This reaction was carried out at RT for $7 \mathrm{hr}$. White solid; Yield $24 \%$; $\mathrm{mp} 220-222{ }^{\circ} \mathrm{C}$; IR $(\mathrm{KBr}) \mathrm{v}_{\max }$ in $\mathrm{cm}^{-1}: 3422(\mathrm{NH}), 3336,3271(\mathrm{CH}$ stretch aromatic), 2930 (CH stretch aliphatic), $1657(\mathrm{C}=\mathrm{O}), 1593\left(\mathrm{NH}\right.$ bend), $1516\left(\mathrm{CH}_{2}\right.$ bend), $1388(\mathrm{C}-\mathrm{F}), 1317(\mathrm{C}-\mathrm{N}), 746(\mathrm{CH}$ bend); ${ }^{1} \mathrm{H}-\mathrm{NMR}$ (DMSO-d $\mathrm{d}_{6}$ ) $\delta$ ppm: 3.17 (t, $2 \mathrm{H}, J=8.1 \mathrm{~Hz}$ ), 4.27 (t, $2 \mathrm{H}, J=8.4 \mathrm{~Hz}$ ), $6.99(\mathrm{t}, 1 \mathrm{H}, J=7.2 \mathrm{~Hz}), 7.15(\mathrm{t}, 1 \mathrm{H}, J$ $=8.1 \mathrm{~Hz}), 7.32(\mathrm{~d}, 1 \mathrm{H}, J=7.2 \mathrm{~Hz}), 7.36(\mathrm{~d}, 2 \mathrm{H}, J=8.7 \mathrm{~Hz}), 7.47(\mathrm{t}, 1 \mathrm{H}, J=7.8 \mathrm{~Hz}), 7.56-7.63(\mathrm{~m}, 1 \mathrm{H}), 7.71(\mathrm{~d}, 2 \mathrm{H}, J=8.5$ $\mathrm{Hz}), 7.76-7.83(\mathrm{~m}, 2 \mathrm{H}), 8.15(\mathrm{~d}, 1 \mathrm{H}, J=8.4 \mathrm{~Hz}), 9.75(\mathrm{~s}, 1 \mathrm{H}), 10.33(\mathrm{~s}, 1 \mathrm{H}) ;{ }^{13} \mathrm{C}-\mathrm{NMR}\left(\mathrm{DMSO}-\mathrm{d}_{6}\right) \delta \mathrm{ppm}: 26.7,53.2,114.5$, $116.0,118.3,120.3,123.8,125.1,126.3,130.5,130.6,133.7,135.5,135.8,137.2,143.0,160.7,163.1$, 163.9, 178.4 ; LCMS calculated for $\left(\mathrm{C}_{22} \mathrm{H}_{18} \mathrm{FN}_{3} \mathrm{OS}\right) \mathrm{m} / \mathrm{z}[\mathrm{M}+\mathrm{H}]^{+} 391.12$, found $\mathrm{m} / \mathrm{z} 392.0887$

\subsubsection{Synthesis of 2-(2-fluorophenyl)-N-(4-(indoline-1-carbothio amido) phenyl)acetamide (5c)}

Prepared as reported above for $5 \mathrm{a}$, starting from compound 2 and 2 -fluoro phenylacetic acid. This reaction was carried out at RT for $7 \mathrm{hr}$. Yellow solid; Yield $24 \% ; \mathrm{mp} 220-222{ }^{\circ} \mathrm{C} ; \mathrm{IR}(\mathrm{KBr}) \mathrm{V}_{\max }$ in $\mathrm{cm}^{-1}: 3328(\mathrm{NH}$ stretch), 3289,3066 (CH stretch, aromatic), 2632 ( $\mathrm{CH}$ stretch, aliphatic), $1672(\mathrm{C}=\mathrm{O}), 1600$ (NH bend), $1510\left(\mathrm{CH}_{2}\right.$ bend), $1388(\mathrm{C}-\mathrm{F}), 1317(\mathrm{C}-$ $\mathrm{N}), 744\left(\mathrm{CH}\right.$ bend); ${ }^{1} \mathrm{H}-\mathrm{NMR}$ (DMSO-d $)$ $\delta \mathrm{ppm}: 3.10(\mathrm{t}, 2 \mathrm{H}, J=8.4 \mathrm{~Hz}), 3.73(\mathrm{~s}, 2 \mathrm{H}), 4.25(\mathrm{t}, 2 \mathrm{H}, J=8.4 \mathrm{~Hz}), 6.97(\mathrm{t}, 1 \mathrm{H}$, $J=6.6 \mathrm{~Hz}), 7.11-7.21(\mathrm{~m}, 3 \mathrm{H}), 7.27(\mathrm{~d}, 4 \mathrm{H}, J=6.8 \mathrm{~Hz}), 7.31-7.42(\mathrm{~m}, 1 \mathrm{H}), 7.54(\mathrm{~d}, 2 \mathrm{H}, J=8.6 \mathrm{~Hz}), 8.16(\mathrm{~d}, 1 \mathrm{H}, J=8.3$ $\mathrm{Hz}$ ), 9.69 (s, 1H), 10.23 (s, 1H) ; ${ }^{13} \mathrm{C}-\mathrm{NMR}\left(\mathrm{DMSO}_{\mathrm{d}}\right.$ ) $\delta \mathrm{ppm:} 26.7,36.2,53.1,115.1,116.0,118.9,122.9,123.0,124.1$, $125.1,126.3,128.7,128.7,131.9,133.6,135.2,143.0,159.4,161.8,167.8,178.4$; LC-MS calculated for $\left(\mathrm{C}_{23} \mathrm{H}_{20} \mathrm{FN} \mathrm{N}_{3} \mathrm{OS}\right)$ $\mathrm{m} / \mathrm{z}[\mathrm{M}+\mathrm{H}]^{+} 405.13$, found $\mathrm{m} / \mathrm{z} 406.0980$.

\subsubsection{Synthesis of $\mathrm{N}$-(4-(indoline-1-carbothio amido) phenyl)cyclopentanecarboxamide} (5d)

Prepared as reported above for $5 \mathrm{a}$, starting from compound 2 and cyclopentane carboxylic acid. This reaction was carried out at RT for $8 \mathrm{hr}$. White solid; Yield $27 \%$; m 213-215 ${ }^{\circ} \mathrm{C}$; IR (KBr) $\mathrm{V}_{\max }$ in $\mathrm{cm}^{-1}: 3298$ (NH stretch), 3040 (CH stretch, aromatic), 2928, 2860 ( $\mathrm{CH}$ stretch, aliphatic), $1670(\mathrm{C}=\mathrm{O}), 1602\left(\mathrm{NH}\right.$ bend), $1518\left(\mathrm{CH}_{2}\right.$ bend), $1317(\mathrm{C}-\mathrm{N})$, 744 (CH bend); ${ }^{1} \mathrm{H}-\mathrm{NMR}\left(\mathrm{DMSO}_{\mathrm{d}}\right) \mathrm{d}$ ppm: 1.56-1.748 (m, 7H), $1.84(\mathrm{~d}, 2 \mathrm{H}, J=8.1 \mathrm{~Hz}), 3.10(\mathrm{t}, 2 \mathrm{H}, J=8.1 \mathrm{~Hz}), 4.25(\mathrm{t}$, $2 \mathrm{H}, J=8.4 \mathrm{~Hz}), 6.98(\mathrm{t}, 1 \mathrm{H}, J=7.5 \mathrm{~Hz}), 7.13(\mathrm{t}, 1 \mathrm{H}, J=7.5 \mathrm{~Hz}), 7.27(\mathrm{~d}, 3 \mathrm{H}, J=8.4 \mathrm{~Hz}), 7.55(\mathrm{~d}, 2 \mathrm{H}, J=8.7 \mathrm{~Hz}), 8.16(\mathrm{~d}$, $1 \mathrm{H}, J=8.1 \mathrm{~Hz}), 9.70(\mathrm{~s}, 1 \mathrm{H}), 9.82(\mathrm{~s}, 1 \mathrm{H}) ;{ }^{13} \mathrm{C}-\mathrm{NMR}\left(\mathrm{DMSO}_{-} \mathrm{d}_{6}\right) \delta \mathrm{ppm}: 26.1,27.2,30.5,45.7,53.6,116.5,119.4,123.43$, 125.6, 125.8, 126.8, 134.1, 135.4, 136.8, 143.6, 174.6, 178.9 ; LC-MS calculated for $\left(\mathrm{C}_{21} \mathrm{H}_{23} \mathrm{~N}_{3} \mathrm{OS}\right) \mathrm{m} / \mathrm{z}[\mathrm{M}+\mathrm{H}]^{+} 365$, found $\mathrm{m} / \mathrm{z} 366.1384$.

\subsubsection{Synthesis of $\mathrm{N}$-(4-(indoline-1-carbothio amido) phenyl)acetamide (5e)}

To a soluction of compound $2(0.4 \mathrm{~g}, 0.00150 \mathrm{~mol})$ in $10 \mathrm{~mL}$ of THF, triethylamine $(0.757 \mathrm{~mL}, 0.0075 \mathrm{~mol})$ was added at $0^{\circ} \mathrm{C}$ followed by acetyl chloride was added. The reaction mixture was allowed to RT and stirred for $1 \mathrm{hr}$. The reaction was monitored by TLC. The reaction mass was concentrated under reduced pressure and separated between ethyl acetate $(50 \mathrm{~mL})$ and water $(50 \mathrm{~mL})$. The combined ethyl acetate layer was washed with $2 \mathrm{~N} \mathrm{HCl}(1 \times 25 \mathrm{~mL}), 10 \%$ $\mathrm{NaHCO}_{3}(2 \times 20 \mathrm{~mL})$, brine $(2 \times 50 \mathrm{~mL})$ and dried over anhydrous $\mathrm{Na}_{2} \mathrm{SO}_{4}$. The ethyl acetate layer was concentrated under reduced pressure to afford compound $5 \mathrm{e}$, white solid; Yield $42 \% \mathrm{mp} 200-202{ }^{\circ} \mathrm{C}$; IR $(\mathrm{KBr}) \mathrm{v}_{\max }$ in $\mathrm{cm}^{-1}: 3428(\mathrm{NH}$ stretch), 3303, 3044 (CH stretch, aromatic), 2928 (CH stretch, aliphatic), $1661(\mathrm{C}=\mathrm{O}), 1600$ ( $\mathrm{NH}$ bend), $1517\left(\mathrm{CH}_{2}\right.$ bend), $1315(\mathrm{C}-$ $\mathrm{N}), 743\left(\mathrm{CH}\right.$ bend); ${ }^{1} \mathrm{H}-\mathrm{NMR}\left(\mathrm{DMSO}_{6} \mathrm{~d}_{6}\right) \delta \mathrm{ppm}: 2.21(\mathrm{~s}, 3 \mathrm{H}), 3.10(\mathrm{t}, 2 \mathrm{H}, \mathrm{J}=8.4 \mathrm{~Hz}), 4.25(\mathrm{t}, 2 \mathrm{H}, \mathrm{J}=8.4 \mathrm{~Hz}), 6.9(\mathrm{t}, 1 \mathrm{H}, \mathrm{J}$ $=7.5 \mathrm{~Hz}), 7.14(\mathrm{t}, 1 \mathrm{H}, \mathrm{J}=7.5 \mathrm{~Hz}), 7.27(\mathrm{~d}, 3 \mathrm{H}, \mathrm{J}=8.7 \mathrm{~Hz}), 7.52(\mathrm{~d}, 2 \mathrm{H}, \mathrm{J}=8.7 \mathrm{~Hz}), 8.16(\mathrm{~d}, 1 \mathrm{H}, \mathrm{J}=8.5 \mathrm{~Hz}), 9.67(\mathrm{~s}, 1 \mathrm{H})$, $9.94(\mathrm{~s}, 1 \mathrm{H}) ;{ }^{13} \mathrm{C}-\mathrm{NMR}\left(\mathrm{DMSO}-\mathrm{d}_{6}\right) \delta \mathrm{ppm}: 23.9,26.5,116.0,118.8,122.9,125.4,126.3,133.6,134.9,136.1,143.0$, 168.0, 178.4 ; LC-MS calculated for $\left(\mathrm{C}_{17} \mathrm{H}_{17} \mathrm{~N}_{3} \mathrm{OS}\right) \mathrm{m} / \mathrm{z}[\mathrm{M}+\mathrm{H}]^{+} 311.11$, found $\mathrm{m} / \mathrm{z} 312.0977$.

\subsection{PHARMACOLOGY}

\subsubsection{In vitro anti-inflammatory activity (Anti-denaturation assay)}

The experiment was carried out with small modification [25]. The standard drug and synthesized compounds were dissolved in minimum quantity of dimethyl Formamide (DMF) and diluted with phosphate buffer (0.2 M, PH 7.4). Final concentration of DMF in all solution was less than $2.5 \%$. Test solution (4 ml) containing different concentrations of drug was mixed with $1 \mathrm{ml}$ of $1 \mathrm{mM}$ albumin solution in phosphate buffer and incubated at $37^{\circ} \mathrm{C}$ in incubator for 15 min. Denaturation was induced by keeping the reaction mixture at $70^{\circ} \mathrm{C}$ in water bath for $15 \mathrm{~min}$. After cooling, the turbidity was measured at $660 \mathrm{~nm}$. Percentage of inhibition of denaturation was calculated from control where no drug was added. The diclofenac sodium was used standard drug. The percentage inhibition of denaturation was calculated by using the following formula.

$$
\% \text { of Inhibition }=(\text { At }- \text { Ac) } \times 100 / \text { At }
$$

At $=$ O.D. of test solution

$A c=$ O.D. of control

\section{RESULTS AND DISCUSSION}

\subsection{Chemistry}

The synthetic scheme for the preparation of intermediates and final compounds are shown in scheme (1-3). The intermediate-1 was prepared [26] by using the reagent 4-nitrophenyl isothiocyanate. The FT-IR spectrum of intermediate-1 
had characteristic NH absorption peaks at $3434 \mathrm{~cm}^{-1}$ and $3300 \mathrm{~cm}^{-1}$. The absorption peaks for $\mathrm{C}=\mathrm{S}$ seen at $1270 \mathrm{~cm}^{-1}$. The $\mathrm{NO}_{2}$ stretching frequency observed at $1319 \mathrm{~cm}^{-1}$ and $1532 \mathrm{~cm}^{-1}$. In the ${ }^{1} \mathrm{H}-\mathrm{NMR}$ spectra of compound-I, the proton signal of thiourea $\mathrm{NH}$ was observed at $\delta 10.39(1 \mathrm{H}$, singlet). Characteristic peaks of thiocarbamide compound were observed at $\delta 10.39 \mathrm{ppm}$. In ${ }^{13} \mathrm{C}$-NMR of intermediate-2, the characteristic peaks at thiocarbamide $\mathrm{C}=\mathrm{S}$ resonate at 177.5 $\mathrm{ppm}$. The intermediate-2 was prepared [27] by reduction of intermediate-1 by $\mathrm{SnCl}_{2}$. The FT-IR spectra of intermediate-2 showed absorption band at $3398 \mathrm{~cm}^{-1}$ due to $\mathrm{NH}$ stretching vibration from resulting amine group formed. In the FT-IR spectrum of intermediate-2, the reduction of $\mathrm{NO}_{2}$ group was confirmed by disappearance of $\mathrm{NO}_{2}$ stretching vibration at $1319 \mathrm{~cm}^{-1}$ and $1532 \mathrm{~cm}^{-1}$. In the ${ }^{1} \mathrm{H}-\mathrm{NMR}$ spectra of intermediate-2, the characteristic amine proton peak seen as broad singlet at $\delta 5.05 \mathrm{ppm}$. The resulting compound was also confirmed by TLC, which was exhibited ninhydrin activity. The intermediate- 2 was taken a common scaffold for synthesis of proposed indoline derivatives. The synthetic path way for intermediate- 2 is outlined below.

\section{Scheme-1}

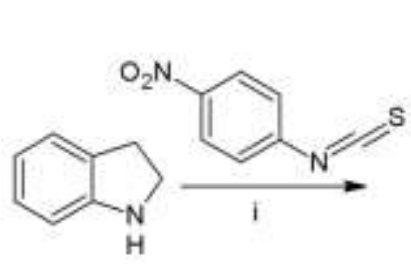<smiles>C/C=C\C=C1\CCN(C(N)=O)C1</smiles>

1

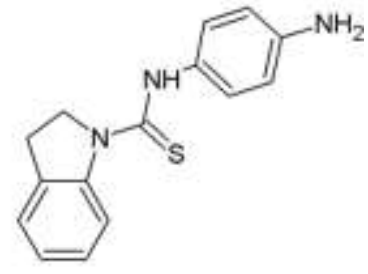

Scheme.1. Reagents and conditions: (i). 4-nitrophenyl isothiocyanate, THF, $0{ }^{\circ} \mathrm{C}$; $12 \mathrm{~N} \mathrm{HCl}, \mathrm{RT}, 0^{\circ} \mathrm{C}$.

(ii) $\mathrm{SnCl}_{2}$

The sulfonamide derivates $4 \mathrm{a}$ and $4 \mathrm{~b}$ were prepared [28] by reaction between corresponding sulfonyl chloride and key intermediate-2. The reacton was carried out in dichloroethane with pyridine base at $90^{\circ} \mathrm{C}$. The FT-IR spectrum resulted in sulfonamide derivatives exhibited absorption wave length at $1154 \mathrm{~cm}^{-1}$ and $1387 \mathrm{~cm}^{-1}$. Which is the characteristic peak of $(\mathrm{S}=\mathrm{O})$ group. The ${ }^{1} \mathrm{H}-\mathrm{NMR}$ spectra of synthesized sulfonamide derivatives showed new singlet peak at $\delta 9.63 \mathrm{ppm}$.

The desired title compounds $\mathbf{3 a}$ and $\mathbf{3 b}$ were obtained in resonable yield [29] by treating key intermediate-2 with corresponding isocyanate and isothiocyanate respectively. In the FT-IR spectrum of 3a characteristic $\mathrm{C}=\mathrm{S}$ seen at 1211 $\mathrm{cm}^{-1}$ while in $3 \mathbf{b}$ characteristic $\mathrm{C}=\mathrm{O} 1649 \mathrm{~cm}^{-1}$. In the ${ }^{1} \mathrm{H}-\mathrm{NMR}$ of $3 \mathrm{a}, \mathrm{NH}$ proton of thiourea group resonated at $\delta 9.78 \mathrm{ppm}$. In the case of $3 \mathrm{~b}, \mathrm{NH}$ proton of new urea group resonated at $\delta 9.67 \mathrm{ppm}$. According to ${ }^{13} \mathrm{C}-\mathrm{NMR}$ of $3 \mathrm{a}$ and $3 \mathrm{~b}$, the compounds has displayed characteristic thiourea carbonyl carbon $\mathrm{C}=\mathrm{S}$ at $\delta 179.9 \mathrm{ppm}$ and urea carbonyl carbon $\mathrm{C}=\mathrm{O}$ at $\delta$ $159.6 \mathrm{ppm}$. The synthetic scheme for preparation of sulfonaminde, urea and thiourea derivatives are shown in Scheme-2.
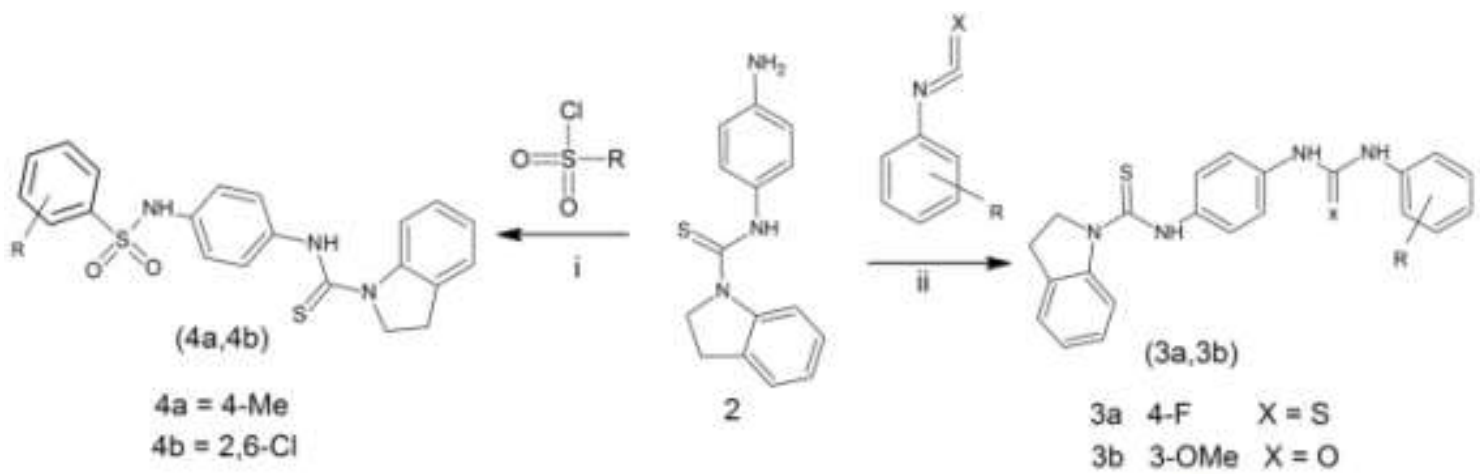

Scheme-2 Reagents and conditions (i). Pyridine, Dichloroethane, $90^{\circ} \mathrm{C}$. (ii). THF, $0^{\circ} \mathrm{C}$.

From the above key intermediate-2, the carboxamide derivatives (5a-d) were prepared[30] by using reagent 1 . Ethyl -3-(3-dimethylaminopropyl) carbodiimide (EDCI). The corresponding acid chloride was used for preparing [31] the amide derivatives $5 \mathrm{e}$. The FT-IR spectrum of all the carboxamide derivatives has strong $\mathrm{C}=\mathrm{O}$ absorption, which showed wave length between $1657-1672 \mathrm{~cm}^{-1}$. The ${ }^{1} \mathrm{H}-\mathrm{NMR}$ spectra of all carboxamide derivatives exhibited broad singals at $\delta$ 9.7-10.1 ppm is assigned to $\mathrm{NH}$. In addition, ${ }^{13} \mathrm{C}-\mathrm{NMR}$ spectra of all carboxamide derivatives displayed signals at $\delta 163.9$ $174.6 \mathrm{ppm}$. All the FT-IR, ${ }^{1} \mathrm{H}-\mathrm{NMR}$ and mass spectral data of compounds $(5 \mathrm{a}-\mathrm{e})$ were in accordance with the proposed molecular structures. The purity of synthesized compounds was monitored by TLC. The synthetic scheme for the preparation of all carboxamide derivatives are depicated in Scheme-3. 


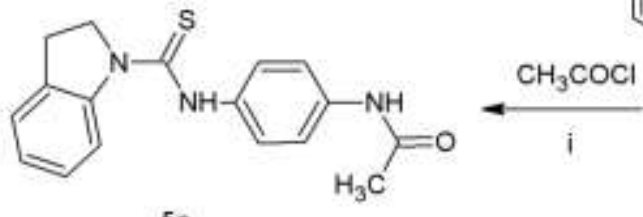

$5 e$

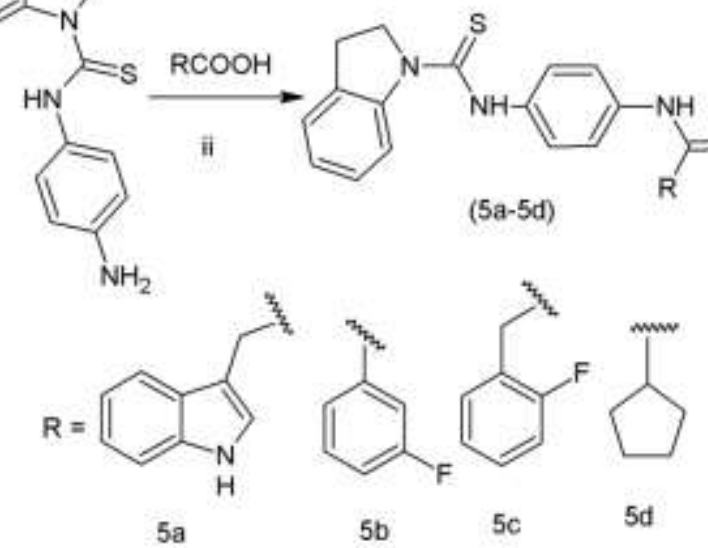

Scheme-3. Reagents and conditions (i). TEA, MDC, $0^{\circ} \mathrm{C}$., (ii). EDCI, HOBt, TEA, THF, $0^{\circ} \mathrm{C}$;

\subsection{Anti-inflammatory activity}

The anti-inflammatory activities of all the above indoline derivatives were examined by standard anti-denaturation assay. The inhibition efficiency of all synthesized indoline derivatives were tested at a concentration ranging from $50 \mu \mathrm{g} / \mathrm{ml}$ to $250 \mu \mathrm{g} / \mathrm{ml}$. The percentage of inhibition and $\mathrm{IC}_{50}$ values of all indoline derivatives are listed in Table 1 . The antidenaturation activity study related that the indoline derivatives $4 \mathrm{a}\left(\mathrm{IC}_{50}=62.2 \mu \mathrm{g} / \mathrm{ml}\right)$ and $4 \mathrm{~b}\left(\mathrm{IC} \mathrm{C}_{50}=60.7 \mu \mathrm{g} / \mathrm{ml}\right)$ showed excellent inhibition activity that is comparable with standard drug diclofenac sodium $\left(\mathrm{IC}_{50}=54.2 \mu \mathrm{g} / \mathrm{ml}\right)$. Furthermore, in the carboxamide derivatives particularly compound $5 \mathrm{a}\left(\mathrm{IC}_{50}=97.8 \mu \mathrm{g} / \mathrm{ml}\right)$ showed moderate anti-denaturation activity. The derivatives $3 \mathrm{a}\left(\mathrm{IC}_{50}=115.4 \mu \mathrm{g} / \mathrm{ml}\right)$ and $3 \mathrm{~b}\left(\mathrm{IC}_{50}=109.1 \mu \mathrm{g} / \mathrm{ml}\right)$ were found to be significant anti-denaturation activity. The compounds $5 \mathrm{c}, 5 \mathrm{~d}$ and $5 \mathrm{e}$ did not show noticeable activity.

Table 1. Anti-inflammatory activity of indoline derivatives (anti-denaturation assay)

\begin{tabular}{|c|c|c|c|c|c|c|}
\hline \multirow{2}{*}{ compound } & \multicolumn{5}{|c|}{$\%$ inhibition } & \multirow{2}{*}{$\begin{array}{c}\mathrm{IC}_{50} \\
(\mu \mathrm{g} / \mathrm{ml})\end{array}$} \\
\hline & $50 \mu \mathrm{g} / \mathrm{ml}$ & $100 \mu \mathrm{g} / \mathrm{ml}$ & $150 \mu \mathrm{g} / \mathrm{ml}$ & $200 \mu \mathrm{g} / \mathrm{ml}$ & $250 \mu \mathrm{g} / \mathrm{ml}$ & \\
\hline $3 a$ & 33.3 & 52.7 & 58.6 & 65.3 & 70.4 & 115.4 \\
\hline $3 b$ & 35.4 & 53.1 & 60.1 & 64.9 & 69.7 & 109.1 \\
\hline $4 a$ & 45.3 & 60.1 & 68.3 & 80.7 & 86.9 & 62.2 \\
\hline $4 b$ & 44.5 & 61.1 & 69.3 & 81.2 & 86.3 & 60.7 \\
\hline $5 a$ & 39.2 & 51.6 & 61.3 & 70.6 & 81.8 & 97.8 \\
\hline $5 b$ & 17.3 & 27.5 & 45.3 & 59.4 & 64.7 & 180.1 \\
\hline $5 c$ & 30.4 & 43.7 & 55.2 & 60.3 & 67.8 & 142.5 \\
\hline $5 d$ & 19.2 & 30.4 & 45.6 & 58.4 & 64.3 & 177.4 \\
\hline $5 e$ & 19.8 & 27.4 & 40.3 & 51.1 & 60.7 & 198.8 \\
\hline $\begin{array}{l}\text { Diclofenac } \\
\text { sodium }\end{array}$ & 47.3 & 61.8 & 70.6 & 81.5 & 90.4 & 54.2 \\
\hline
\end{tabular}

The in vitro anti-inflammatory study revelated that the synthesized final compound, containg sulfonamide group showed very good inhibition on protein denaturation. Furthermore, carboxamide based final compound bearing indole acetic acid group showed significant activity when comparing with other carboxamide based derivatives. Urea and thiourea based compounds showed noticeable inhibition on protein denaturation, which is less than sulphonamide derivatives.

The synthesized of compounds $\mathbf{4 a}$ and $\mathbf{4 b}$ could offer an excellent framework that may lead to the discovery of new potent anti-inflammatory agents. 


\section{CONCLUSION}

It could be concluded that compounds $\mathbf{4 a}$ (Synthesis of $\mathrm{N}$-(4-(tosylamino) phenyl) indoline-1-carbothio amide) and $\mathbf{4 b}$ (Synthesis of $\mathrm{N}$-(4-\{[(2,6-dichlorophenyl)sulfonyl ]amino\}phenyl) indoline-1-carbothioamide) showed potent inhibition on protein denaturation. Based on the above findings the novel indoline derivatives $\mathbf{4 a} \mathbf{a} \mathbf{4 b}$ selected for future optimisation and development in animal models for detailed efficacy studies.

\section{ACKNOWLEDGEMENT}

The authors are very much thankful to Dr.R.S.D. Wahida Banu, Principal, Government College of Engineering Salem-11 and gratefully acknowledged.

\section{REFERENCES}

[1] Chevan, P., Mane, A .S. and Shingare, M.S. 2001. Indian j Chem, 408, 339.

[2] Adel, A., Gendy, E.I. and Aly, Ahmedy, M. 2000. Arch Pharm Res, 23, 310- 314.

[3] Sureyya Olgena, and Semiha Ozkanb, 2009. Naturforsch, 64,155- 162.

[4] Abdel Rahman, A.H, Keshk, E.M, Hanna, M.A. and Sh, El Bady M., 2004. Bioorganic\&Medicinal Chemistry,12, 2483- 2488.

[5] Girija, S., Singh, Patrick Luntha, 2009. European Journal of Medicinal Chemistry, 44, 2265-2269.

[6] Irena Sovic Sandra Kraljevic PavelicElitza Markova Carb, Natasa llic Raja Nhilic, Sabine Depauw, Marie Helene David Cordonnier., and Grace Karminski Zamola, 2014, European Journal of Medicinal Chemistry,87,372-385.

[7] Pengzhan Li, Yanmei Tan, Guyue Liu, Yang Liu, Jianzhen Liu, Yanzhen Yin, Guisen Zhao, 2014, Drug Discoveries \& Therapeutics,8, 110-116.

[8] Xiao Liang Xu, Chun Lei Yu, Wen Chen, Ying Chao Li, Juan Yang Li, Yan Li, Hong BinZhang, Xiao Dong Yang, 2015, Org Biomol Chem,13,1550-1557.

[9] Kang Jin, Xiaopan Zhang, Chunhun Ma, Yingying Xu, Yumei Yuan, Wenfang Xu, 2013, Bioorganic \& Medicinal Chemistry,21, 2663-2670.

[10] Jae Hwan Kwak, Yoseob Kim, Hyunjeong Park, Jae Yong Jang, Keun Kuk Lee, Wonhui Yi,Jeong Ah Kwak, Song Gyu Park, Hwanmook Kim, Kiho Lee, Jong Soon Kang, Sang Bae Han, 2010, Bioorganic \& Medicinal Chemistry Letter,.20, 4620-4623.

[12] Kenjiro Sato, Hiromichi Sugimoto, Kentaro Rikimaru, Hiroshi Imoto, Masahiro Kamaura, Nobuyuki Negoro, Yoshiyuki Tsujihata, Hirohisa Miyashita, Tomoyuki Odani, Toshiki Murata, 2014, Bioorganic \& Medicinal Chemistry, 22,1649-1666.

[13] Svetiana Furman, Elinor Nissim Bardiugo, Shani Zeeli, Michal Weitman, Abraham Nudelman, Efrat Finkin Gorner, Dorit Moradov, Helena Shifrin, Donna Schorer Apelbaum, Marta Weinstock, 2014, Bioorganic \& Medicinal Chemistry Letter,24, 2283-2287.

[14] Rajanarendar, E., Ramakrishna, S., Govardhan Reddy, Nagaraju, D., Reddy, Y.N., 2013, Bioorganic \& Medicinal Chemistry Letters, 23, 3954-3958.

[15] Jang Yang Chang, Hsing Pang Hsieh, Chi Yen Chang, Kuo Shun Hsu, Yi Fang Chiang, Chi Ming Chen, Ching Chuan Kuo and Jing Ping Liou, 2006, J Med Chem,49, 6656-6659.

[16] Shoji Kamiya, Hiroaki Shirahase, Akihisa Yoshimi, Shohei Nakamura, Mamoru Kanda, Hiroshi Matsui, Masayasu Kasai, Kenji Takashi and Kauyoshi Kurahashi, 2000, Chem Pharm Bull,48, 817-827.

[17] Tetsuji Noguchi, Naoki Tanaka, Toyaki Nishimata, Riki Goto, Miho Hayakawa, Atsuhiro Sugidachi, Taketoshi Ogawa, Fumitoshi Asai, Tomoko Ozeki and Kochi Fujimoto, 2007, Chem Pharm Bull, 55, 393-402.

[18] Li Sun, Ngoc Tran, Flora Tang, Hrald App, Peter Hirth, Gerald Mcmahon., and Cho Tang, 1998, J Med Chem,41,2588-2603.

[19] Kenji Takahashi, Masayasu Kasai, Masaru Ohta, Yoshimichi Shoji, Kazuyoshi., and Hiroaki Shirahase, 2008, J Med Chem,51,4823-4833.

[20] Tomihiro Nishiyama, Tatsuya Suzuki,Yasuhiro Hashiguchi, Shingo Shiotsu, Masataka Fujioka, 2002, Polymer Degradation and Stability, $75,549-554$.

[21] Wagdy, M., Mohamed Fares, Hany, S., Ibrahim, Mohamed, H., Aly, Suher Zada, Mamdouh, M., Alid, Saher, M., Abou Seri, Hatem, Abdel Aziz, Dalal, A., Abou El Ella, 2015, European Journal of Medicinal Chemistry, 100, 89-97.

[22] Samir Mahndiratta, Yi Fang Chiang, Mei Jung Lai, Hsueh Yun Lee, Mei Chuan Chen, Ching Chuan Kuo, Chi Yen Chang, Jang Chang, Jing Ping Liou, 2014, Bioorganic \& Medicinal Chemistry, 22, 4917-4923. 
[23] He Zhao, Xiaoshu He, Andrew Thurkauf, Diane Hoffman, Andrzej Kieltyka, Robbin Brobeck, Renee Primus., and Jan W F Wasley, 2002, Bioorganic \& Medicinal Chemistry Letters,12, 3111-3115.

[24] Ramakrishna V S Nirogi, Rajesh Kumar Badange, Kiran Kumar Kandukuri and Mukkanti Khagga, 2015 J.Chem. Sci.127 439445 .

[25] Dan Zhang, Hao Song and Young Qin, 2011 Accounts of chemical research. 44447457.

[26] Gnana Ruba Priya, Girija and Ravichandran N, 2011 Rasayan J of Chemistry, 4418424.

[27] Sidoova E, Kralova K and Loss D, 1998 Molecules 3135.

[28] Kartizky AR and Rachwal S, 1994 J.Hetrocyclic Chem. 31775.

[29] Benigno M, Isabel G, Maria VV, Joaquin B and Francisca S 2002 Polyhedron 211229.

[29] Zhili X, Hongyu Z, Michael S, Bruce GS, Harriet TS, Tom SS and Rich SJ,2008 Bioorganic \& Medicinal Chemistry Letters.18 4298.

[30] Jing X, Hai Feng Z, Ya Juan Z, Yun Jun L, Ji Wang J, Jing Jing Y and Shu Feng Z, 2009 Molecules 143142.

[31]Seung Woo Y, Hee Yeol L, Bong Hwan C, Kyung Mi A, Jung Su R, Young Ho L and Jae Hoon K, 2006 Bull.korean chem.Soc 277. 